Zwei Jahre nachdem das Zentrum für Holocaust-Studien am Institut für Zeitgeschichte eingerichtet wurde, zieht sein Leiter, Frank Bajohr, eine erste Bilanz. Mit einer Serie internationaler Konferenzen und Aufenthalten zahlreicher Gastwissenschaftler ist bereits eine starke Vernetzung in der internationalen Forschercommunity erreicht worden. Eine ganze Reihe von Publikationen wurde erarbeitet, und das Zentrum gibt gezielte Impulse für die universitäre Lehre. München, das mit seiner außerordentlichen Dichte von historischen Forschungseinrichtungen und Gedenkstätten beste Rahmenbedingungen für seine Arbeit bietet, ist aus dem Adressbuch der internationalen Holocaustforschung nicht mehr wegzudenken.

Frank Bajohr

\title{
Zwei Jahre Zentrum für Holocaust-Studien am Institut für Zeitgeschichte
}

\section{Ausgangslage und Zielsetzungen}

Im Jahre 2010 kursierte am US Holocaust Memorial Museum in Washington (USHMM) ein Papier mit dem Titel „Holocaust-Forschung in Deutschland - eine Geschichte ohne Zukunft?“". Es beklagte eine „rasche Erosion der nie hinreichend verankerten wissenschaftlichen Infrastruktur", da es in Deutschland keine dem Center for Advanced Holocaust Studies in Washington oder dem International Institute for Holocaust Research in Jerusalem vergleichbare Einrichtung gebe. Zudem existiere in Deutschland - anders als in den USA, Großbritannien oder in Israel - kein einziger universitärer Lehrstuhl, der sich schwerpunktmäßig der Geschichte des Holocaust widme. Resümierend hieß es in dem Papier: „Das Problem ist das Fehlen institutioneller Bedingungen in Deutschland, die die HolocaustForschung auf Dauer zum festen Bestandteil des Wissenschaftslebens machen. Wer heute in der Bundesrepublik zum Holocaust forscht, tut dies mehr als in den letzten zwanzig Jahren in thematischer Isolation, ohne feste universitäre Anbindung und mit geringer Aussicht auf beruflichen Erfolg. “2

Nicht zu Unrecht wurde beklagt, dass den in den USA, Israel und vielen europäischen Ländern im Bereich der Holocaust-Forschung tätigen Einrichtungen ausgerechnet in Deutschland ein institutioneller Partner fehle und ein Äquivalent zu den Förder- und Fellowship-Programmen in USA und Israel hierzulande nicht existiere.

\footnotetext{
${ }^{1}$ Eine ausführliche Version des Papiers wurde publiziert von Jürgen Matthäus, Holocaust-Forschung in Deutschland. Eine Geschichte ohne Zukunft?, in: Michael Brenner/Maximilian Strnad (Hrsg.), Der Holocaust in der deutschsprachigen Geschichtswissenschaft. Bilanz und Perspektiven, Göttingen 2012, S. 27-41.

${ }^{2}$ Unveröffentlichtes Manuskript, im Besitz des Verfassers.
} 
Nach Anregung durch Wendy Lower (Claremont McKenna College, USA) installierte das Institut für Zeitgeschichte auf Betreiben seines Direktors, Andreas Wirsching, und in Kooperation mit den Lehrstuhlinhabern für Neueste Geschichte und Zeitgeschichte sowie jüdische Geschichte und Kultur an der LudwigMaximilians-Universität München (LMU), Margit Szöllösi-Janze und Michael Brenner, 2013 ein Zentrum für Holocaust-Studien, zunächst in einer bis Ende 2016 terminierten Vorlaufphase, um so die Schaffung eines größer dimensionierten Zentrums mit drei zentralen Arbeitsschwerpunkten schrittweise vorzubereiten:

Erstens soll das Zentrum eine international attraktive Forschungsinfrastruktur mit Fellowships für Gastwissenschaftler ${ }^{3}$ entwickeln, um Doktorandinnen und Doktoranden, Post-Docs und auch Spitzenforschern einen Ort der Forschung und Kommunikation bereitzustellen, der zugleich den engen wissenschaftlichen Austausch der deutschen mit der internationalen Holocaust-Forschung gewährleistet.

Zweitens soll das Zentrum die Forschung über den Holocaust in Deutschland vorantreiben und dabei eng mit internationalen Forschungseinrichtungen kooperieren. Obwohl die Geschichte dieses präzedenzlosen Jahrhundertverbrechens auf absehbare Zeit einen besonderen Stellenwert in der deutschen Geschichte einnehmen wird, hat die deutsche Forschung in den letzten Jahren klassische nationale Perspektiven überwunden und in bemerkenswerter Weise grenzüberschreitende Perspektiven und Arbeitskontakte entwickelt. Langfristig wird ein international ausgeschriebenes Stipendienprogramm für Doktoranden einerseits die Holocaust-Forschung in Deutschland institutionell sichern und diese andererseits eng mit der weltweiten Forschung verzahnen. Dabei soll nicht zuletzt eine Kooperation zwischen dem Zentrum bzw. dem Institut für Zeitgeschichte und der LMU München eine enge Verbindung von Forschung (insbesondere gemeinsamen Promotions- und Postdoc-Programmen) und Lehre gewährleisten.

Drittens schließlich soll das Zentrum für Holocaust-Studien in Zusammenarbeit mit der LMU München die universitäre Lehre über die Geschichte des Holocaust sicherstellen und darüber hinaus allgemein Lehrende ermuntern, entsprechende Lehrveranstaltungen an deutschen Universitäten anzubieten: durch Publikationen, die das zunehmend komplexere Forschungsfeld für Lehrende, Lernende und eine interessierte Öffentlichkeit erschließen, aber auch durch Veranstaltungen für Hochschullehrerinnen und Hochschullehrer, die einen systematischen Erfahrungsaustausch der oft isoliert arbeitenden Lehrenden ermöglichen sollen.

In seiner Vorlaufphase wird das Zentrum in erster Linie durch Mittel des Bundesministeriums für Bildung und Forschung getragen und soll schließlich in eine Bund-Länder-Finanzierung überführt werden. Mit lediglich zwei vollen Wissenschaftler-Stellen (Frank Bajohr, Leiter und Andrea Löw, stellv. Leiterin), einer weiteren, im Rahmen des europäischen EHRI-Projektes ${ }^{4}$ finanzierten Stelle (Giles

\footnotetext{
${ }^{3}$ Aus Gründen besserer Lesbarkeit bezeichnet hier und im gesamten Text das generische Maskulinum Männer und Frauen gleichermaßen.

${ }^{4}$ European Holocaust Research Infrastructure, vgl. www.ehri-project.eu; siehe auch S. $144 \mathrm{ff}$.
} 
Bennett) sowie je zwei wissenschaftlichen und studentischen Hilfskräften hat das Zentrum im Sommer 2013 seine Vorlaufphase begonnen, sich aber dennoch schnell als wichtige Forschungseinrichtung im Bereich der Holocaust-Studien etabliert. Die ersten zwei Jahre seines Bestehens sollen zum Anlass genommen werden, die bisherige Tätigkeit zu bilanzieren und zugleich nach Perspektiven der weiteren Arbeit zu fragen. Im Folgenden können die wichtigsten Aufgabenfelder nur kurz umrissen werden. So bleiben beispielsweise die zahlreichen Aktivitäten in Öffentlichkeit und Medien (Vorträge, Interviews, Beteiligung an Gedenkveranstaltungen, wissenschaftliche Expertisen vor Gericht ${ }^{5}$ ) unerwähnt, die aufzuzählen den Rahmen dieses Beitrages sprengen würde.

\section{Internationale Konferenzen}

Schon im ersten Jahr seines Bestehens hat das Zentrum drei internationale Konferenzen durchgeführt bzw. vorbereitet und sich damit als Ort wissenschaftlichen Austausches über den Holocaust etabliert. Am Anfang stand ein in Verbindung mit der Akademie für Politische Bildung in Tutzing organisierter Workshop (April 2014), auf dem führende Holocaust-Forscher aus aller Welt eine kritische Zwischenbilanz verschiedener Forschungsansätze zur Geschichte des Holocaust zogen, Möglichkeiten seiner historischen Kontextualisierung diskutierten und über Desiderate künftiger Forschung debattierten ${ }^{6}$. Alle wesentlichen Ergebnisse des Workshops wurden in einem Band der „Schwarzen Reihe“ des S. Fischer Verlages für ein breiteres Publikum zusammengefasst. Er erschien 2015 und eignet sich in besonderer Weise als einführende Publikation für Lehrveranstaltungen zur Geschichte des Holocaust ${ }^{7}$.

Die nächste Konferenz machte einen wesentlichen Interessen- und Arbeitsschwerpunkt des Zentrums deutlich, nämlich eine Gesellschaftsgeschichte des Holocaust, die sich darum bemüht, eine statische Zuschreibung im Sinne der klassischen Trias „Täter-Opfer-Bystander“ zu überwinden: Vom 23. bis 25. Oktober 2014 organisierte das Zentrum in München eine große internationale Tagung (The Holocaust and European Societies. Social Processes and Social Dynamics), auf der sich Wissenschaftler aus vierzehn Ländern mit jenen gesellschaftlichen Prozessen und sozialen Dynamiken in den europäischen Gesellschaften beschäftigten, in die der Holocaust eingebunden war bzw. die durch ihn ausgelöst wur$\operatorname{den}^{8}$. Die Konferenz folgte der Erkenntnis jüngerer Forschungsansätze, dass der Holocaust nicht allein ein ideologisch fundierter und politisch exekutierter Vorgang war, sondern auch ein gesellschaftlicher Prozess, an dem unzählige Personen

\footnotetext{
${ }^{5}$ So fungierte beispielsweise Frank Bajohr 2015 als Sachverständiger im Prozess gegen den SSAngehörigen Oskar Gröning vor dem Landgericht Lüneburg.

${ }^{6}$ Zu Programm und Tagungsbericht siehe: http:/ / hsozkult.geschichte.hu-berlin.de/tagungsberichte $/$ id $=5412 \&$ siew $=$ pdf.

${ }^{7}$ Vgl. Frank Bajohr/Andrea Löw (Hrsg.), Der Holocaust. Ergebnisse und neue Fragen der Forschung, Frankfurt a. M. 2015.

${ }^{8}$ Zu Programm und Tagungsbericht siehe http:/ / hsozkult.geschichte.hu-berlin.de/tagungsberichte $/ \mathrm{id}=5789 \&$ view $=$ pdf.
} 
in den europäischen Gesellschaften als Akteure, partiell auch als Profiteure, beteiligt waren ${ }^{9}$.

An diese Konferenz knüpfte im September 2015 eine weitere Tagung des Deutschland-Instituts der Universität Amsterdam an, bei der das Zentrum als Mitveranstalter fungierte: „Probing the Limits of Categorization. The ,Bystander“ in Holocaust History" ${ }^{10}$ fragte nach der Genese, dem Erkenntnispotenzial, vor allem jedoch nach den Problemen, die für eine europäische Gesellschaftsgeschichte des Holocaust mit der Zuschreibung Bystander verbunden sind - ein Begriff, der im Deutschen oft mit „Zuschauer“ übersetzt wird und eine eher passive Distanz zum Mordgeschehen suggeriert. Damit verfehlt er jedoch jene vielfältige Grauzone sozialen Verhaltens, in der sich Mitmachen, Hinnehmen, Distanzieren und partielles Widerstehen in sehr komplexer Weise mischten.

Über dieses Verhalten im gesellschaftlichen Alltag geben in besonders eindringlicher Weise Tagebuchaufzeichnungen, Briefe und sonstige subjektive Zeugnisse Auskunft. Diese standen im Mittelpunkt des Dachauer Symposiums 2014, deren wissenschaftliche Leitung Frank Bajohr übernommen hatte, zusammen mit Sybille Steinbacher, die seit 2013 die Projektleitung der Dachauer Symposien innehat. Die Publikation zur Tagung erschien $2015^{11}$. Im gleichen Jahr veröffentlichten das Zentrum für Holocaust-Studien und die Arbeitsstelle Holocaustliteratur der Universität Gießen eines der eindrucksvollsten subjektiven Zeugnisse, das in einem Ghetto unter deutscher Besatzungsherrschaft entstanden ist: die auf Jiddisch verfassten Tagebuchaufzeichnungen von Jósef Zelkowicz aus dem Ghetto Litzmannstadt, die dieser im September 1942 unter dem Eindruck einer mehrtägigen Massendeportation verfasst hatte ${ }^{12}$.

Zwei Tagungen im Jahre 2015, die das Zentrum für Holocaust-Studien und das Mandel Center for Advanced Holocaust Studies des US Holocaust Memorial Museum in Washington organisierten, zeugten von der internationalen Vernetzung des Zentrums und zugleich von der besonders intensiven deutsch-amerikanischen Kooperation in diesem Forschungsfeld: Im Juli 2015 gab ein „Teaching Summit“ Lehrenden an deutschen Universitäten, die Lehrveranstaltungen über den Holocaust anbieten, erstmals Gelegenheit zu einem umfassenden Erfahrungsaustausch. Eine ausführliche Analyse der Lehrpraxis an deutschen Universitäten ergab, dass entsprechende Lehrveranstaltungen in verschiedenen Disziplinen durchaus flächendeckend angeboten werden, mancherorts aber nur jeweils von einem einzelnen Lehrenden pro Universität. Zudem beruht dies ausschließlich auf jeweils persönlicher Initiative, da das Thema Holocaust faktisch nirgendwo in bestehenden universitären Programmen verankert ist, zum Beispiel in den Mas-

\footnotetext{
${ }^{9}$ Ein Konferenzband wird im Jahre 2016 im Verlag Palgrave MacMillan erscheinen.

${ }^{10}$ Zum Programm siehe: http://duitslandinstituut.nl/the-abystandera-in-holocaust-history.

${ }^{11}$ Vgl. Frank Bajohr/Sybille Steinbacher (Hrsg.), „...Zeugnis ablegen bis zum letzten“. Tagebücher und persönliche Zeugnisse aus der Zeit des Nationalsozialismus und des Holocaust, Göttingen 2015.

${ }^{12}$ Vgl. Jósef Zelkowicz, In diesen albtraumhaften Tagen. Tagebuchaufzeichnungen aus dem Getto Lodz/Litzmannstadt, September 1942, hrsg. von Angela Genger, Andrea Löw und Sascha Feuchert, Göttingen 2015.
} 
ter-Studiengängen. Darauf ist von außen kaum Einfluss zu nehmen, doch besteht zweifellos ein Bedürfnis nach besserer Vernetzung der Lehrenden und einem regelmäßigen Erfahrungsaustausch, dem das Zentrum nach seinem weiteren Ausbau ab 2017 Rechnung tragen will.

Im Oktober 2015 veranstalteten Zentrum und USHMM schließlich eine Tagung, die sich der Forschung an deutschen Gedenkstätten und NS-Dokumentationszentren widmete. Forschung steht in der Regel nicht im Mittelpunkt der zumeist pädagogisch-didaktischen Arbeit von Gedenkstätten. Oft ist sie eher lokal ausgerichtet, was bisweilen auch ihre Wahrnehmung im nationalen und internationalen Rahmen begrenzt. Dies soll sich ebenfalls auf mittlere Sicht ändern, haben doch viele Forschungen ein Potenzial, das weit über die jeweilige Gedenkstätte hinausweist.

Der osteuropäische Raum ist für die Holocaust-Forschung von besonderer Bedeutung, wenngleich Forschung und Lehre dort nur schwach institutionell verankert sind. Um die wissenschaftlichen Kontakte in Richtung Osteuropa zu pflegen, hatten sich deshalb IfZ und Zentrum im November 2013 an einer internationalen Konferenz beteiligt, die von Prof. Il'ja Al'tman (Russian Research and Educational Holocaust Center, Moskau) in Kaliningrad organisiert wurde und an die Novemberpogrome 1938 erinnerte: „Lessons of the Holocaust and Contemporary Russia. Marking 75 years after the Kristallnacht“. Im Juni 2015 setzte sich diese Kooperation fort, als mehrere Referenten von IfZ und Zentrum (u. a. Jürgen Zarusky, Frank Bajohr und Andrea Löw) an der Konferenz „Lessons of the Holocaust and Contemporary Russia“ in Moskau teilnahmen.

\section{Fellowship-Programm}

Forschungen zum Holocaust haben sich in den letzten zwei Jahrzehnten so stark internationalisiert, dass genuin nationale Perspektiven zwar keineswegs verschwunden sind, aber doch merklich an Bedeutung eingebüßt haben. Im Generationswandel haben sich einstmals dominierende nationale Gedächtniskollektive abgeschwächt. $\mathrm{Zu}$ dieser Entwicklung hat die Internationalisierung der Forschungsdiskussion maßgeblich beigetragen, nicht nur befördert durch internationale Konferenzen, sondern auch durch Fellowships für Gastwissenschaftler, die anfänglich vor allem in Washington und Jerusalem angeboten wurden und den Austausch der Forscher aus den verschiedenen Ländern vorangetrieben haben. Ein solches Angebot hat in Deutschland bislang gefehlt, und deshalb gehören Fellowships für ausländische Gastwissenschaftler auch zu den infrastrukturellen Kernaufgaben des Zentrums für Holocaust-Studien. Das Institut für Zeitgeschichte und das Zentrum für Holocaust-Studien haben im Zeitraum von 2013 bis 2015 insgesamt vierzehn Fellows bei ihrem Forschungsaufenthalt in München betreut ${ }^{13}$. Unter ihnen befanden sich nicht wenige Gastwissenschaftler

\footnotetext{
${ }^{13}$ Im Einzelnen waren folgende Fellows am IfZ - Zentrum für Holocaust-Studien tätig: Dana Smith, Queen Mary University of London (Jüdischer Kulturbund in Bayern 1934-1938); Froukje Demant, Universität Amsterdam (The Daily Relations of Jews and Non-Jews in the
} 
aus Osteuropa, die in ihren jeweiligen Ländern in einem politisch wie wissenschaftlich bisweilen schwierigen Umfeld arbeiten müssen. Auf diese Weise kommt dem Zentrum auch geografisch eine wichtige Brückenfunktion nach Osteuropa $\mathrm{zu}$.

Darüber hinaus hat das Zentrum zusammen mit dem Mandel Center for Advanced Holocaust Studies am US Holocaust Memorial Museum ein Joint Fellowship eingerichtet, um den wechselseitigen deutsch-amerikanischen Austausch von Wissenschaftlern im Bereich der Holocaust-Geschichte besonders zu fördern ${ }^{14}$. Schließlich fungierte das Zentrum als Gastgeber für ausländische Fellows, denen ein Forschungsaufenthalt in München im Rahmen des europäischen EHRI-Programmes ermöglicht wurde ${ }^{15}$. Allen Fellows bot sich in München ein ideales Gesprächsumfeld. Sie konnten ihre Projekte im Wissenschaftlichen Kolloquium des Instituts für Zeitgeschichte präsentieren, wobei einige der Präsentationen in Kooperation mit dem Oberseminar des Lehrstuhls für Jüdische Geschichte der Ludwig-Maximilians-Universität München stattfanden. Zum anregenden Gesprächsklima trug nicht allein die fachwissenschaftliche Expertise des Zentrums für Holocaust-Studien bei, sondern auch die große Zahl von Wissenschaftlerinnen und Wissenschaftlern, die im Institut für Zeitgeschichte zu Themen der NS-Geschichte forschen.

\section{European Holocaust Research Infrastructure (EHRI)}

Im Jahre 2011 startete ein zentrales EU-finanziertes Projekt, das Strukturen ausbauen und vertiefen soll, um die europäischen Forschungs- und Archivressourcen zur Geschichte des Holocaust dauerhaft zu vernetzen. Mittlerweile ist als Abschluss der ersten Projektphase ein online-Portal verfügbar, das die weltweit beste

German-Dutch Border Region 1925-1955); Yurii Radchenko, Universität Charkiv, Ukraine (Ukrainian Hilfspolizei, Self-Government, and the Holocaust in Ukraine); Elisabeth Pönisch, Universität Freiburg (,Judenhäuser“ im Deutschen Reich ab 1939); Diana Dumitru, Ion Creangă State Pedagogical University of Moldova (Traumatic Encounters: Jews, Gentiles and the Soviet State in the Aftermath of the Holocaust); Aleksander Kruglov, Ukrainian Institute for Holocaust Studies, Dnepropetrovsk (The Holocaust in the USSR Regions Occupied by Germans. Problem of Regional Features and Periodization); Felix Mattheis, Universität Hamburg („Hamburg im Osten“. Die Besatzung Polens und der Holocaust aus der Perspektive der Hansestadt 1939-1945); Adam Gellert, Central European University, Budapest (Partners in Crime: The German-Hungarian Solution of the Jewish Question in Hungary in 1944).

${ }^{14}$ In den Jahren 2013 und 2014 waren im Rahmen des Joint Fellowship zu Gast: Tom Frydel, University of Toronto (Polish „Blue Police“ in the Holocaust) und Sari Siegel, University of Southern California (Medicine Behind Barbed Wire: Jewish Prisoner-Physicians in Nazi Labor, Concentration and Extermination Camps); 2016 wird zu Gast sein: Natalia Aleksiun, Touro College, New York (Daily Survival. Social History of Jews Hiding in Eastern Galicia).

${ }^{15}$ Als EHRI-Fellows waren zu Gast: Devra Katz, University of Haifa (Emotions in Stutthof. An Analysis of the Social Function of Emotions in a Prisoner Society), Aleksandra Loewenau, Oxford Brookes University (GB) (Rebuilding Lives of Jewish Survivors of Medical Experiments at Auschwitz. A Comparative Study), Katarzyna Person, Jewish Historical Institute Warsaw (Polish-Jewish Relations in Germany in the Immediate Postwar Period) und Matt Lawson, Edge Hill University (GB) (Film Music of German Holocaust Cinema). 
Informationsressource über Archivbestände zum Holocaust darstellt ${ }^{16}$. Für die Jahre 2015-2019 hat die Europäische Kommission mit einer Fördersumme von acht Millionen Euro ein Fortsetzungsprojekt bewilligt, das von 23 Forschungseinrichtungen aus 15 europäischen Staaten, Israel und den USA getragen wird. Im Rahmen des EHRI-Projektes, das gegenwärtig in 14 Arbeitsschwerpunkte („Work Packages") aufgeteilt ist, haben das Institut für Zeitgeschichte bzw. das Zentrum für Holocaust-Studien im Project Management Board verantwortlich mitgearbeitet und u.a. zwei Work Packages geleitet: WP 4 (Coordinating Transnational Access to Research Infrastructures, Leitung bis August 2013: Johannes Hürter, danach Frank Bajohr) und WP 5 (Training, Leitung: Andrea Löw). Koordiniert wurden die EHRI-Aktivitäten von IfZ und Zentrum durch Giles Bennett. Damit waren IfZ und Zentrum für die Organisation der EHRI-Fellowships (an fünf Standorten), für die Online-Kurse zur Geschichte des Holocaust sowie für die Summer Schools des EHRI-Programmes zuständig. Im Rahmen der Online-Kurse hat das Zentrum eine Kurseinheit über „Ghettos Under Nazi Rule“ (Konzeption: Andrea Löw und Giles Bennett) sowie über „The Germans and the Holocaust“ (Konzeption: Sonja Schilcher) konzipiert und mit den „Country Reports“17 eine mehr als zweihundert Seiten umfassende Informationsressource zu europäischen Institutionen, Archiven und Archivbeständen über den Holocaust in 47 verschiedenen, vor allem europäischen Ländern erarbeitet (Mitherausgeber: Pascal Trees und Giles Bennett). Für die bedeutsame polnische Archivlandschaft wurde darüber hinaus der wichtigste Archivführer zu entsprechenden Beständen komplett ins Englische übersetzt und aktualisiert; er wird im Internet der wissenschaftlichen Öffentlichkeit zur Verfügung gestellt ${ }^{18}$. Im EHRI-Portal finden sich gegenwärtig Informationen über 1.800 Archive in 57 Ländern, in über 460 Institutionen sind über 150.000 Holocaust-bezogene archivische Einheiten nachgewiesen.

Internationale Summer Schools für jüngere Wissenschaftler haben im Rahmen des EHRI-Programmes in Paris, Amsterdam, Jerusalem und München stattgefunden, wobei Mitarbeiter des Zentrums an allen Veranstaltungen als Referenten beteiligt waren. Die „Summer School on Holocaust Research“ in München (22.7. 9. 8. 2013) fand in der Akademie für Politische Bildung in Tutzing statt und wurde verantwortlich von Andrea Löw und Giles Bennett organisiert. Diese Summer School für zwölf europäische Nachwuchswissenschaftler war mit der „Münchner Internationalen Sommeruniversität“ („MISU Summer School“) für zwölf junge Forscherinnen und Forscher aus den USA verbunden. Diese gemeinsam vom Zentrum und Wendy Lower (Claremont McKenna College, USA) konzipierte und mit der LMU München durchgeführte Munich Summer School erwies sich als großer Erfolg. In einem intensiven Lehrplan, an dem sich prominente Forscher wie

\footnotetext{
${ }^{16}$ Näheres ist der offiziellen Homepage unter www.ehri-project.eu zu entnehmen.

17 Vgl. http://www.ehri-project.eu/national-reports.

${ }^{18}$ Alina Skibińska, Guide to the Sources on the Holocaust in Occupied Poland http://training.ehri-project.eu/sites/default/files/portal_assets/skibinska_guide.pdf; Mitherausgeber: Giles Bennett und Pascal Trees.
} 
Christopher Browning und Alan Steinweis beteiligten, wurden Methoden und Tendenzen der internationalen Holocaustforschung vermittelt.

\section{Forschung}

Trotz des eindeutigen Tätigkeitsschwerpunktes in der Entwicklung der Forschungs-Infrastruktur hat das Zentrum für Holocaust-Studien auch in der Forschung bereits Akzente gesetzt. Das wichtigste Projekt bildete die Edition der politischen Tagebücher des NS-Chefideologen und Reichsministers für die besetzten Ostgebiete, Alfred Rosenberg, aus den Jahren 1934-1944. Die Tagebücher wurden 2013 im verstreuten Nachlass von Robert Kempner, dem amerikanischen Ankläger im Nürnberger Prozess, durch Mitarbeiter des US Holocaust Memorial Museums entdeckt und vom Mandel Center for Advanced Holocaust Studies des Museums (Jürgen Matthäus) und dem Zentrum für Holocaust-Studien (Frank Bajohr) gemeinsam herausgegeben. Für die Geschichte des Holocaust sind die Tagebücher Rosenbergs, die um bislang unbekannte Dokumente aus dem Nachlass Rosenbergs ergänzt werden, insofern von großer Bedeutung, als sie die Frage nach den wesentlichen politischen Entscheidungsprozessen und namentlich nach Rosenberg als Mitverantwortlichem des Holocaust aufwerfen und auf der Basis der neu aufgefundenen Quellen auch beantworten. Jürgen Matthäus und Frank Bajohr schrieben eine mehr als 120seitige Einführung, das Tagebuch selbst wurde unter Beteiligung der wissenschaftlichen Hilfskräfte des Instituts mit zahlreichen kommentierenden Fußnoten erschlossen. Die deutsche Ausgabe der Edition erschien $2015 \mathrm{im}$ S. Fischer Verlag ${ }^{19}$. Noch im gleichen Jahr kamen eine französische, eine englisch-amerikanische und eine spanische Ausgabe auf den Markt, wobei letztere sogar Platz 7 der spanischen Bestseller-Liste erreichte ${ }^{20}$. Eine polnische und eine portugiesische Ausgabe sind in Vorbereitung. Darüber hinaus hat das Zentrum für Holocaust-Studien ein Forschungsprojekt über „Diplomatic Reports on the Persecution of Jews and the Holocaust in Europe" konzipiert. Es knüpft an ein 2010/11 durchgeführtes Projekt über die Berichterstattung ausländischer Diplomaten aus dem Dritten Reich an, das die diplomatischen Berichte von zehn Ländern vergleichend analysierte ${ }^{21}$. Das neu konzipierte Projekt soll demgegenüber bislang nicht berücksichtigte Länder in Ost- und Nordeuropa (Tschechoslowakei, Ungarn, Rumänien, Bulgarien, Schweden, Finnland etc.) erfassen und thematisch auf Berichte über Judenverfolgung und den Holocaust fokussiert werden. Wie sein Vorläufer kann das Projekt nur mit einem Netzwerk

\footnotetext{
${ }^{19}$ Vgl. Jürgen Matthäus/Frank Bajohr (Hrsg.), Alfred Rosenberg. Die Tagebücher 1934-1944, Frankfurt a. M. 2015.

${ }^{20}$ Vgl. Alfred Rosenberg, Journal 1934-1944, ed. par Jürgen Matthäus et Frank Bajohr, Paris 2015; Jürgen Matthäus/Frank Bajohr, The Political Diary of Alfred Rosenberg and the Onset of the Holocaust, Lanham/MD. 2015; Alfred Rosenberg, Diarios 1934-1944, Edición a cargo de Jürgen Matthäus y Frank Bajohr, Barcelona 2015.

${ }^{21}$ Vgl. Frank Bajohr/Christoph Strupp (Hrsg.), Fremde Blicke auf das „Dritte Reich“. Berichte ausländischer Diplomaten über Herrschaft und Gesellschaft in Deutschland 1933-1945, Göttingen 2011.
} 
von Historikern aus den beteiligten Ländern verwirklicht werden, die mit der Quellenüberlieferung in ihren Ländern besonders vertraut sind. Von daher ist das EHRI-Projekt, das die für die Geschichte des Holocaust relevanten Quellenbestände in Europa erschließt, ein besonders geeigneter Rahmen für ein solches internationales Projekt, das als Teil des Work Package 12 gebilligt und bewilligt wurde, sodass es im Zeitraum von 2015-2019 realisiert werden kann.

Schließlich hat das Zentrum eine Reihe von Einzelforschungen angeregt und auf den Weg gebracht, die hier nicht im Detail aufgeführt werden können. Zu den wichtigsten Projekten gehört das Promotionsvorhaben von Anna-Raphaela Schmitz über „Rudolf Höß - Handlungspraxis, Beziehungsstrukturen und Privatleben eines KZ-Kommandanten“. Es unternimmt den Versuch, eine Biografie des Auschwitz-Kommandanten Rudolf Höß vor allem aus „praxeologischer“ Perspektive zu schreiben und sein Handeln nicht primär durch vermeintliche biografische Prägungen und Kontinuitäten zu erklären, sondern vor allem durch eine detaillierte Analyse der Handlungspraxis und ihrer Determinanten im KL Auschwitz, nicht zuletzt der personalen Netzwerke, in die Rudolf Höß eingebunden war. Im Rahmen dieses Projektes wird auch der private Nachlass des Auschwitz-Kommandanten umfassend ausgewertet.

\section{Pläne und Perspektiven}

1) Forschung und Lehre über den Holocaust bedürfen in Deutschland aktiver Zentren wissenschaftlicher Expertise mit nationaler und internationaler Ausstrahlung. Neben Berlin mit seinen Universitäten, Museen, Dokumentationszentren und Forschungseinrichtungen sowie Frankfurt (u.a. Fritz-Bauer-Institut, geplanter Lehrstuhl für Holocaust-Studien an der Johann Wolfgang Goethe-Universität) ist München hier als Standort prädestiniert, da es über zahlreiche Gedenkstätten, Fachbibliotheken, Dokumentationsorte und Archive, vor allem jedoch über eine Dichte einschlägig arbeitender Zeithistoriker am Institut für Zeitgeschichte und an der Ludwig-Maximilians-Universität verfügt, die andernorts kaum vorzufinden ist. München kann und soll deshalb zu einem zentralen Standort für Forschung und Lehre über den Holocaust werden, der die spezifische Expertise von IfZ und Zentrum für Holocaust-Studien mit der Exzellenz der LMU in der Neueren und Neuesten Geschichte, der Osteuropäischen und Jüdischen Geschichte zusammenführt. Mit seinen zahlreichen Akademien und Bildungsträgern bietet sich München überdies als zentraler Standort an, um Fortbildungen und einen Erfahrungsaustausch für Lehrende durchzuführen, die an deutschen Universitäten über den Holocaust unterrichten, aber auch für Mitarbeiter und wichtige Multiplikatoren, die an Gedenkstätten und Dokumentationszentren tätig sind.

2) Bei dem geplanten Ausbau des Zentrums wird sich das Angebot für Gastwissenschaftler am Zentrum für Holocaust-Studien deutlich erweitern, sodass nicht allein Doktoranden und vereinzelte Post-Docs, sondern auch Senior Fellows aufgenommen werden können. Damit liegen auch alle Voraussetzungen vor, das Fellowship-Programm u.a. durch thematisch fokussierte Workshops zu einem 
Forschungskolleg auszubauen. Ähnlich wie mit den USA wird auch mit Israel ein institutionalisierter Austausch von Wissenschaftlern angestrebt, z. B. mit dem Strochlitz Institute for Holocaust Research, das an der Universität Haifa ein internationales MA- und PhD-Programm in Holocaust Studies betreibt.

3) Das Interesse der Forschung hat sich in den letzten Jahren zu Recht vermehrt dem osteuropäischen Raum zugewandt, ohne dass die vorhandenen Quellenbestände bislang auch nur annähernd ausgewertet worden sind, z. B. die der zahlreichen Sonderkommissionen und Gerichte, die der Roten Armee im Verlauf des Krieges westwärts folgten und vor allem gegen Kollaborateure und Beteiligte des Holocaust vorgingen. Es mangelt wahrlich nicht an griffigen Thesen über „Gewalträume“ in Osteuropa, in denen der Raum bisweilen selbst zum Akteur avanciert und Gewalt selbsterklärend, nämlich als struktureller Selbstlauf einmal entfesselter Gewalt, definiert wird ${ }^{22}$, oder an Behauptungen über angebliche Interdependenzen von nationalsozialistischem und stalinistischem Terror in den so genannten „Bloodlands“23. Es fehlt auch nicht an monokausalen Zuspitzungen wie die der mangelnden Staatlichkeit im osteuropäischen Raum als zentralem Erklärungselement für die Praxis des Holocaust ${ }^{24}$. Es mangelt jedoch entschieden an empirischen Studien, die jenseits luftiger Thesen den Holocaust genauer in soziale Prozesse und Interaktionen unter deutscher Besatzungsherrschaft in Osteuropa einzuordnen und mit zentralen Leitvorstellungen des NS-Regimes zu verbinden wissen. Dies wird in den nächsten Jahren zu den wichtigsten Forschungsschwerpunkten des Zentrums gehören.

4) In Zukunft wird München als Konferenzort und Ort wissenschaftlicher Diskussion um den Holocaust weiter an Bedeutung gewinnen. Im Februar 2016 veranstaltet das Zentrum für Holocaust-Studien eine internationale Tagung, die sich mit einem für die Vorgeschichte des Holocaust zentralen Thema befasst: mit dem Aufstieg rechtspopulistischer, autoritärer und faschistischer Regime in Europa in den 1930er Jahren und der antisemitischen Politik und Praxis am Vorabend des Holocaust. Die Tagung steht unter der Überschrift „Europe 1935-1941. RightWing Politics and the Rise of Antisemitism“25. Über einzelne thematische Konferenzen hinaus besteht in Europa ein zusätzlicher Bedarf an institutionalisierten Konferenz- und Gesprächszusammenhängen, wie sie in den USA vor allem durch die zweijährlich stattfindenden Lessons \& Legacies-Konferenzen hergestellt werden, die sich als „Historikertag“ der Holocaustforscher etabliert haben, jedoch hauptsächlich von nordamerikanischen Wissenschaftlern besucht werden. Wegen hoher Flugkosten und Konferenzgebühren sind sie vor allem für jüngere, nicht zuletzt osteuropäische Forscher kaum erreichbar, sodass es sinnvoll wäre, im Jahr

\footnotetext{
${ }^{22}$ Vgl. Jörg Baberowski/Gabriele Metzler (Hrsg.), Gewalträume. Soziale Ordnungen im Ausnahmezustand, Frankfurt a. M. 2012.

${ }^{23}$ Vgl. Timothy Snyder, Bloodlands. Europa zwischen Hitler und Stalin, München 2010.

${ }^{24}$ Vgl. ders., Black Earth. Der Holocaust und warum er sich wiederholen kann, München 2015.

${ }^{25}$ Die Tagung vom 18.-20. 2. 2016 wird vom Zentrum gemeinsam mit Dieter Pohl (Alpen-AdriaUniversität Klagenfurt) und Grzegorz Krzywiec (Instytut Historii PAN, Warschau) veranstaltet.
} 
zwischen den amerikanischen Konferenzen mit einer „Lessons \& Legacies Munich" einen europäischen Ableger zu etablieren.

5) Deutsche Historikerinnen und Historiker sind vor allem in den letzten 25 Jahren an der weltweiten Intensivierung der Forschung über den Massenmord an den europäischen Juden beteiligt gewesen und haben grundlegende Forschungsbeiträge verfasst, die freilich nicht überall gleichermaßen rezipiert wurden. Vor allem im nicht-anglophonen Ausland - zum Beispiel unserem Nachbarland Frankreich - sind die Ergebnisse der deutschen Forschung oft unbekannt geblieben. Aus diesem Grunde sollen im Jahre 2017 zwei Sonderbände der Révue d'histoire de la Shoah unter dem Arbeitstitel „Die deutsche Geschichtsschreibung und die Shoah. Entwicklung, Themen und Ergebnisse seit 1990“ in Kooperation zwischen dem Memorial de la Shoah und dem Zentrum für Holocaust-Studien veröffentlicht werden. Im Rahmen seiner internationalen Vernetzung kommt dem Zentrum die wichtige Aufgabe zu, die Ergebnisse der deutschen Forschung jenseits der Landesgrenzen besser bekannt zu machen. Umgekehrt besteht in der Bundesrepublik ein wachsendes Bedürfnis nach online-gestützten Informationsangeboten, die die verwirrende Vielfalt internationaler Konferenzankündigungen, ausgeschriebener Fellowships und Stipendien mit grundlegenden Informationenressourcen für die Forschung zusammenführt und auch Möglichkeiten des interaktiven Informationsaustauschs bietet. Dies böte vor allem jenen Forscherinnen und Forschern wichtige Hilfestellungen, die in deutschen Städten und Regionen ohne spezifische wissenschaftliche Expertise in Sachen HolocaustForschung leben. Schließlich ist es Aufgabe des Zentrums, in ganz Deutschland Forschung und Lehre über den Holocaust anzuregen. 